\title{
Role of Transesophageal Echocardiography in the Diagnosis of Multi-chamber Intracardiac Thrombosis During Liver Transplantation: A Case Series
}

\author{
Jocelyn Y. Wang, G. Burkhard Mackensen, Alexander Vitin*, Kenneth Martay \\ University of Washington, Seattle, WA, USA
}

\begin{abstract}
Intra-cardiac thrombosis is one of the most devastating complications during liver transplantation. In the majority of cases, ICT, followed by massive pulmonary embolism, is commonly occurring shortly after liver graft reperfusion, but it has been reported to occur at any stage of the surgery. We present a series of 3 cases of intra-cardiac thrombosis during orthotopic liver transplantation surgery, including a case of four-chamber intra-cardiac clot formation during the pre-anhepatic stage. This article represents a single-centre 14 year-long experience. Intra-operative TEE is the gold standard to diagnose intra-cardiac thrombosis, monitoring its size, location and dynamics, as well as myocardial performance and the effects of resuscitation efforts.
\end{abstract}

Keywords: intra-cardiac thrombosis, liver transplantation, transesophageal echocardiography

Received: 8 May 2020 / Accepted: 23 July 2020

\section{INTRODUCTION}

Substantial perioperative blood loss, pre-existing and on-going coagulopathy and blood transfusion side effects are major concerns in the perioperative management of liver transplant recipients. Also, hypercoagulability and associated risk for intra-cardiac and intra-vascular obstructive clot formation have been recognized as substantial threats for patient and graft survival.

Abnormal thrombus formation mechanisms during liver transplant remain poorly understood, especially in the presence of impaired coagulation factor synthesis and consumption-related deficits in end-stage liver disease (ESLD). Notwithstanding the growing number of reported cases and abundancy of experimental and clinical studies of coagulopathy, both coagulation deficit and hypercoagulable states, the abnormal thrombus formation, resulting in life-threatening flow obstructions, remains mostly unpredictable, let alone preventable.

Intra-cardiac thrombosis (ICT) formation during orthotopic liver transplantation (OLT) is a catastrophic event, frequently leading to a fatal outcome. It may oc- cur at any stage of surgery, with increased incidence in the period immediately following the graft reperfusion. Reported incidence of ICT has been between $0.71 \%=$ $6.25 \%$, with overall mortality rate of $68 \%-82 \%$ intraoperatively $[1,2]$.

A few cases of massive ICT, where obstructive clot material was successfully removed with subsequent patient survival, have been reported [3-5]. However, in the majority of cases, the rapid development of circulatory shock, followed by cardiac arrest due to flow obstruction, almost invariably leads to a poor outcome. Fatal outcomes have been observed despite timely employed intraoperative Trans-esophageal Eco-cardiography (TEE) diagnostics, cardio-pulmonary resuscitation (CPR), use of anticoagulants, thrombolytics, and other treatment options. The hypercoagulability state, resulting in obstructive thrombus formation during liver transplantation, presents a challenge for the anaesthesia provider, and a proper solution is yet to be found.

In this case series, three cases of intracardiac thrombus formation during liver transplantation, presenting a single-centre 14-years -long experience are reported. 


\section{CASE 1}

A 59-year-old female with ESLD secondary to nonalcoholic steatohepatitis (NASH) and a "Model for End-Stage Liver Disease" score (MELD) of 35, was presented for a liver transplant.

The decision to proceed with the liver transplant had been made by the Liver Transplant Selection Committee a few months earlier. As soon as the organ offer was accepted, the patient was admitted to the University of Washington Medical Center, Seattle, WA, USA, about 24 hours before the expected surgery start.

Her past medical history was notable for end-stage renal disease (ESRD) on dialysis due to hepato-renal syndrome, and severe pulmonary hypertension (PHTN).

Her severe PHTN improved significantly with sildenafil citrate tablets, (VIAGRA; ${ }^{\varpi}$ Pfizer, New York, US) for oral use. This resulted in a steady decrease in pulmonary artery systolic pressure (PASP) from 79-84 $\mathrm{mmHg}$ to mid-40s.

Pre-operative trans-thoracic ECO cardiography (TTE) showed a normal left ventricular (LV) size and systolic function with an estimated ejection fraction (EF) of 70\%., moderately elevated PASP $(43-48 \mathrm{~mm}$ $\mathrm{Hg}$ ) based on normal right atrial pressure $(0-5 \mathrm{~mm}$ $\mathrm{Hg}$ ), moderate dilation of RA, and mildly dilated LV with normal systolic function. Laboratory tests showed a hematocrit of $19 \%$, international normalised ratio (INR) 2.1, prothrombin time (PT) 23.3", platelets (PLT) $24,000 / \mu \mathrm{l}$, and fibrinogen (Fib) $121 \mathrm{mg} / \mathrm{dl}$.

During the pre-anhepatic phase, four units of red blood cells (RBCs), two units of fresh frozen plasma (FFP), and one unit of platelets (PLT) were transfused. This resulted in an improvement of coagulation status (INR up to 1.9, PT to 19.2", Fib to $135 \mathrm{mg} / \mathrm{dl}$., PLT up to $43,000 / \mu \mathrm{l}$ ).

A thromboelastogram (TEG) showed signs of coagulation deficit; prolonged $\mathrm{r}$ of $10^{\prime}\left(n: 4-8^{\prime}\right)$ and $\mathrm{K}$ of $8^{\prime}\left(n: 1-4^{\prime}\right)$ - times, (maximum amplitude (MA) of $41 \mathrm{~mm}$ (n:55-73mm). There were no signs of fibrinolysis, lysis percentage $<1.5 \%$, n: $0-8 \%$.

Throughout most of the pre-anhepatic phase, the patient remained stable and inotropic support was not required. About 90 minutes into the pre-anhepatic phase, the patient suddenly developed circulatory collapse, followed shortly after that by cardiogenic shock and cardiac arrest.
CPR was immediately initiated. A TEE was performed and showed ICT in all four cardiac chambers and also within the tricuspid, aortic and mitral valves. After continued CPR for about thirty minutes, the thrombus in the LA and the LV appeared to have cleared. However, it was still visualized in the RA.

The LV shown severe wall motion abnormalities in the inferior and inferior-septal regions, RV functions appeared to be worse when compared to the last TEE; the free wall of the RV was akinetic.

The possibility of acute myocardial infarction (MI), likely in the right coronary artery (RCA) distribution region, was considered. TEE video clips demonstrate clots located in all four chambers (Figure1; Online supplementary video 1); a massive clot in mitral position on the biplane view (Figure 2; Online supplementary video 2); and clots in both ventricles, as demonstrated on Figure 3 (Online supplementary video 3), that also showed that right ventricular outflow tract (RVOT) inflow-outflow was almost obstructed with a clot in AV.

An interventional radiology consultant obtained promptly after the discovery of the clot initially recommended the removal of the thrombus in the interventional radiology suite.

Along with continuous CPR efforts and in preparation for the patient's transport to the interventional radiology suite, Heparin Sodium USP, (Pfizer, NJ, US), 50,000 Units, has been administered intravenously by the anesthesiologist, as per institutional guidelines.

The restoration of effective circulation was unattainable despite the concomitant administration of intravenous epinephrine (Epinephrine, International Medication Systems, Ltd., (IMS), South El Monte, California, US.), norepinephrine bitartrate (Levophed, Bitartart, Pfizer, NJ, US) and phenylephrine hydrochloride (Vazculep, Hikma Pharmaceuticals USA Inc. Eatontown, NJ USA) infusions at high-rates of $1 \mathrm{mcg} /$ $\mathrm{kg} / \mathrm{min}, 1.2 \mathrm{mcg} / \mathrm{kg} / \mathrm{min}$ and $2.4 \mathrm{mcg} / \mathrm{kg} / \mathrm{min}$, respectively, sodium bicarbonate boluses each $50 \mathrm{mEq}$ to a total of $150 \mathrm{mEq}$, epinephrine boluses, $1 \mathrm{mg}$ each to a total of $9 \mathrm{mg}$, given intravenously, and vasopressin (Vasostrict, Sigma Aldrich, St. Louis, MO, US and Germany) boluses, 2-4 units up to a total of 24 units, given intravenously. Continuous CPR was also undertaken concurrently.

Considering the TEE findings, specifically the presence of flow-obstructing clots in all four cardiac chambers and also the absence of spontaneous effec- 


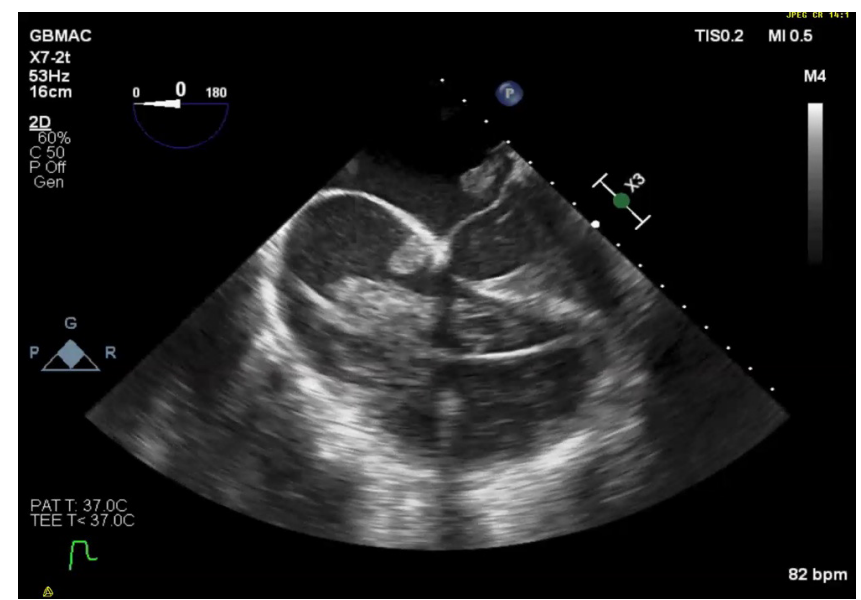

Fig. 1. Clots located in all four chambers

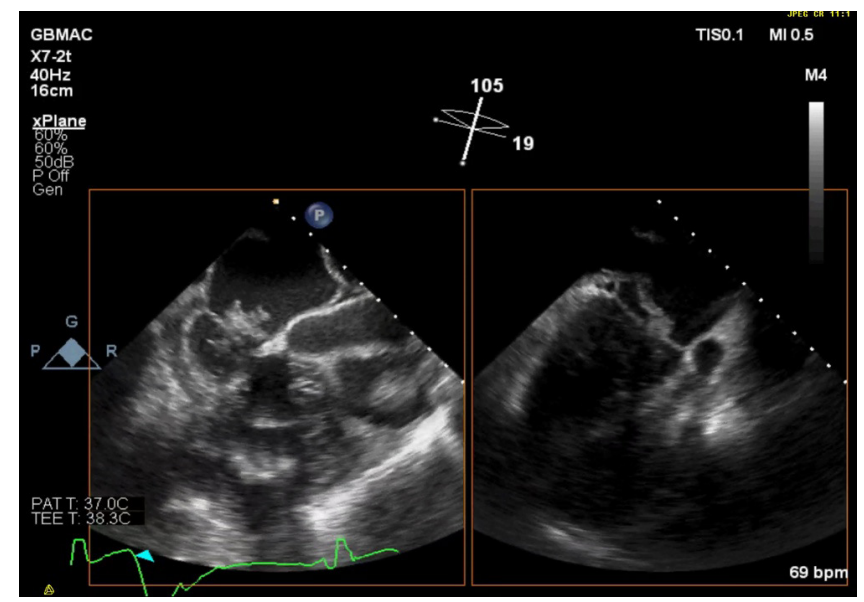

Fig. 2. Massive clot in mitral position on the biplane view

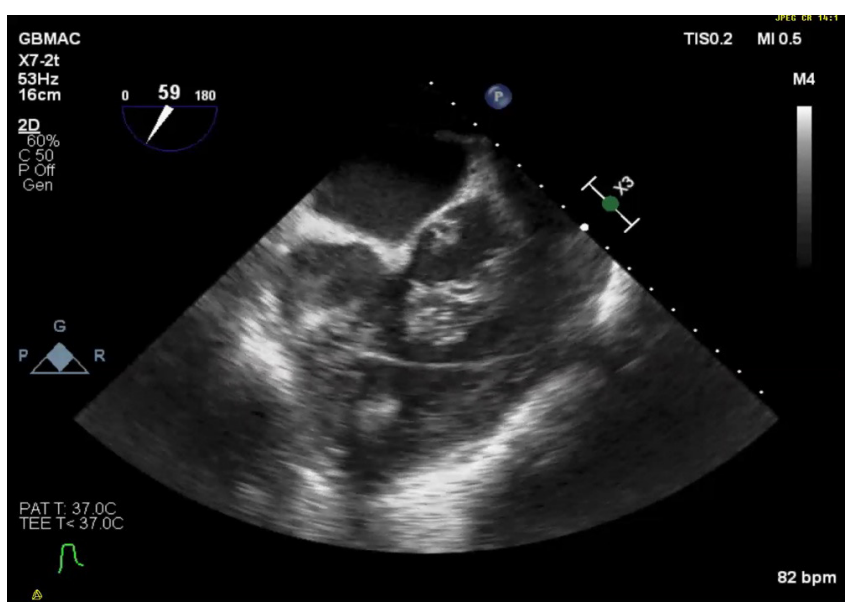

Fig.3 Large clots in the RV outflow position

tive circulation, the transplant surgery was aborted. After consulting again with the interventional radiology team, carrying out the removal of clots was now considered futile, and the decision has been made to transfer the patient to the critical care unit. The patient expired within one hour, despite continuous CPR and vasoactive agents' infusions.

\section{CASE 2}

A 60-year-old female with ESLD secondary to hepatocellular carcinoma (HCC) and hepatitis C, a MELD score of 22, was presented for liver transplant.

The decision to proceed with the liver transplant had been approved by Liver Transplant Selection Committee approximately three months earlier. As soon as the organ offer was accepted, the patient was admitted to the University of Washington Medical Center, Seattle, WA, USA, about 48 hours before the expected surgery start.

Pre-operative TTE demonstrated a normal LV systolic function with an LVEF of $60-70 \%$, and a mild relaxation abnormality. Minimal aortic regurgitation of a normal-appearing aortic valve was detected. The stress ECO showed an appropriate hemodynamic response to pharmacologic stress without evidence of myocardial ischemia.

Baseline laboratory results showed a mild coagulation deficit (INR of 2.2, Fib of $90 \mathrm{mg} / \mathrm{dl}$, PLT of 65,000 / $\mu \mathrm{l}$, Hematocrit (Hct) of 24\%, and baseline TEG showed signs of a mild coagulation deficit ( $\mathrm{r} 8$ 8, $\mathrm{K}$ 6, MA $47 \mathrm{~mm}$, lysis $0.9 \%$ )

During the pre-anhepatic phase, the patient became progressively hemodynamically unstable. While her cardiac output (CO) decreased from baseline 11 to $7-8 \mathrm{~L} / \mathrm{min}$, the systemic vascular resistance (SVR) increased minimally from mid-200 to 430 dynes/seconds $/ \mathrm{cm}^{-5}$ with vasopressors' support. However, the pulmonary artery systolic pressure (PASP) steadily increased from a baseline of 26-32 up to 45-50 mmHg. In anticipation of worsening pulmonary hypertension after graft reperfusion, the decision had_been made to initiate nitric oxide inhalation (INOmax ${ }^{\oplus}$, INOTherapeutics LLC, New Jersey, US) at 25 ppm, as per institutional guidelines.

Approximately 20 minutes into the NO administration, the TEE demonstrated evidence of moderate RV dilation and mild-to-moderate diastolic dysfunction. LV function was deemed unchanged.

Liver graft re-circulation was performed slowly over approximately ten minutes. TEE assessment, performed after attaining hemodynamic stability following the graft reperfusion, approximately ten minutes after portal vein de-clamping, was suggestive of a nonobstructive right atrial clot, taking up to more than $50 \%$ of its volume. 
After completion of the hepatic artery anastomosis, the patient became progressively acidotic and hemodynamically unstable, with rapidly declining arterial blood pressure, and TEE-detected evidence of worsening both RV systolic and diastolic functions.

After obtaining laboratory results, that showed a Hct of 18 and an INR of 2.6, volume status optimization and coagulation deficit correction were undertaken. This the included IV infusion of $500 \mathrm{ml}$ of $5 \%$ albumin (Albumin Human, Mitsubishi Chemical Holdings America Inc. New York NY,\& Virginia VA), and the transfusion of four units of red blood cells, and two units of FFP.

However, due to a rapid decline of myocardial performance, resulting in circulatory collapse, CPR was initiated. Effective perfusion was restored for about eight minutes, but then heart rhythm deteriorated to a terminal slow junctional pattern, followed by an asystolic arrest.

Amidst continuous resuscitation efforts, a pericardiotomy was performed to allow direct manual heart compressions. TEE revealed a large clot, obstructing the right atrium and most of the right ventricle, with a smaller clot in the pulmonary artery, almost completely blocking forward flow (Figure 4; Online supplementary video 4 ).

Despite prolonged open cardiac massage, the myocardial contractions and effective circulation could not be restored, and the patient expired.

\section{CASE 3}

A 48-year-old male with ESLD (MELD score 14) secondary to hepatitis C (HCV)-induced cirrhosis, and

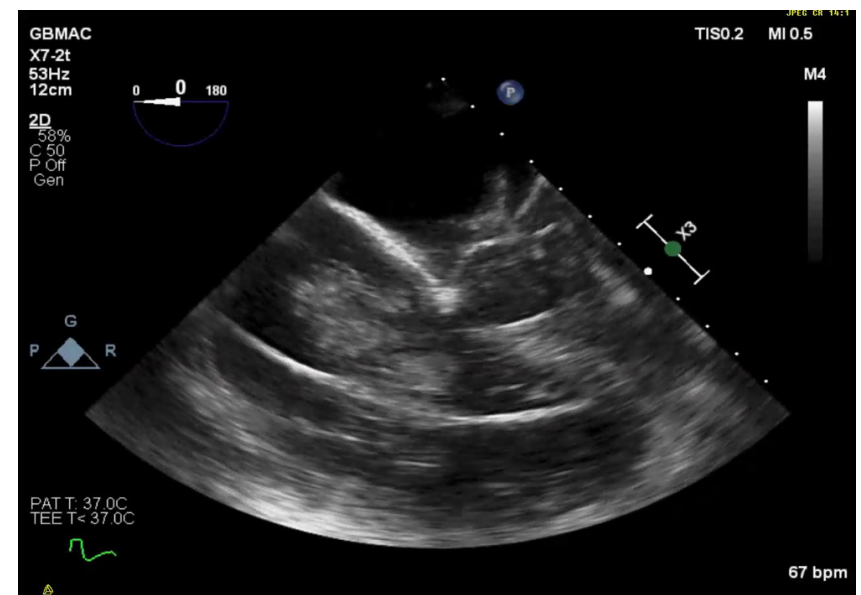

Fig. 4. Large clot, obstructing the right atrium and most of the right ventricle, with a smaller clot in the pulmonary artery hepatocellular carcinoma was admitted for liver transplantation.

The decision to proceed with the liver transplant has been approved by the Liver Transplant Selection Committee less than one month earlier. As soon as the organ offer was accepted, the patient was admitted to the University of Washington Medical Center, Seattle, WA, USA, about forty-eight hours before expected surgery start.

A pre-operative TTE demonstrated a mild LA enlargement and an estimated RV systolic pressure of $30 \mathrm{mmHg}$ which suggested a degree of tricuspid regurgitation. LV functions were within the normal range, EF of $76 \%$. A stress ECO revealed no evidence of myocardial ischemia or infarction.

Baseline laboratory tests showed an international normalized ratio) of 1.9, a fibrinogen concentration of $174 \mathrm{mg} / \mathrm{dl}$, platelets $93,000 / \mu \mathrm{l}$, and a hematocrit of $29 \%$. The pre-anhepatic phase of the surgery was uneventful. However, due to significant sclerosis in the retroperitoneum, the surgeons chose to use a bi-caval technique. The patient tolerated the inferior vena cava (IVC) clamping very well.

After reperfusion of the liver graft, the patient was initially stable. His TEG shown signs of coagulation deficit ( $\mathrm{r}$ and K times 10' and 12', respectively, MA 32 $\mathrm{mm}$, lysis 3.2\%, INR 4.6, Fib $78 \mathrm{mg} / \mathrm{dl}$, PLT 76,000/ $\mu$, HCT of $21 \%$ ), which was corrected with a transfusion of four units of FFP, one unit of cryoprecipitate, one unit of PLT, and three units of RBCs.

After a short while, patient's oxygen saturation dropped into the mid-80s, central venous pressure (CVP ) and the pulmonary artery systolic pressure (PASP) increased from an average of $8 \mathrm{~cm} \mathrm{Hg}$ to $26 \mathrm{~cm}$ $\mathrm{Hg}$ and from 32 to $55 \mathrm{~mm} \mathrm{Hg}$, respectively.

Circulatory collapse ensued. Intraoperative TEE demonstrated massive clots in the RA, RV and the pulmonary artery. RA and RV were severely dilated, and the LV appeared to be empty. Despite all resuscitative efforts, the patient condition continued to deteriorate rapidly. A thoracotomy was performed for direct manual heart compressions. A cardiac surgery consult determined that, given the prolonged downtime and the extensive amount of intracardiac clot, the situation was not salvageable.

After prolonged, albeit unsuccessful CPR, effective circulation could not be restored, and the patient expired. 


\section{DISCUSSION}

Recent studies have suggested, that ESLD may not only be associated with coagulopathy, but also with hypercoagulation. These findings implied that the overall hemostatic abilities of the cirrhotic liver might not be as severely compromised as previously assumed. For example, an almost doubled risk of pulmonary embolism in patients with liver disease compared to the general population was found in a large case-control study [6]. Intricate mechanisms of perioperative hypercoagulability in liver transplant recipients, resulting in major obstructing vascular thrombotic events, are exceedingly complex, and have already been reviewed and discussed in numerous publications $[7,8]$.

Early detection and accurate diagnosis of an intracardiac thrombus - preferably in the process of formation - is critically important for patient survival, as well as in determining the choice and extent of treatment options. TEE provides means both for early diagnosis and monitoring of already settled thrombi, particularly for flow impediment and obstructions. The wide range of reported incidence of ICT $(0.71 \%-6.25 \%$, with an intraoperative mortality rate of $68 \%-82 \%[1,2])$ and ICT-associated mortality may be explained partially by non-universal intraoperative use of TEE during liver transplantation (hence lower likelihood of immediate thrombus detection).

Increased recognition of TEE as an efficient diagnostic/monitoring modality is likely related to the growing number of anaesthesia providers, receiving at least basic training with TEE certifications [9]. Recent surveys have demonstrated the routine use of TEE in only $38 \%$ to $40 \%$ of liver transplant centres, with TEE mostly used during catastrophic hemodynamic instability and resuscitation efforts. There seems to be a gradual increase in TEE-use during anaesthesia for liver transplantation. [10-12]. However, there are groups of researchers, advocating for and against making TEE as a standard monitoring modality for liver transplant anaesthesia care $[13,14]$.

A potential complication of the use of TEE in liver transplants may be haemorrhage, related to the insertion and manipulation of a probe, with an estimated incidence of $0.1-0.3 \%$ in cardiac surgery cases [15]. This may be more common in liver transplant recipients. Another issue with TEE is the potential inaccuracy of TEE- derived estimates of PA pressure, CVP, and CO.

With a relatively high probability of PA pressure fluctuations during portal and IVC clamping, and especial- ly around graft reperfusion, the accurate estimation of it requires a high-quality image and an adequate tricuspid regurgitation. Cywinski J et al. [14] recommended that the Doppler signal must be aligned parallel to the regurgitant jet. This is sometimes difficult to achieve in situations of hemodynamic instability when it is needed the most. On the other hand, PA catheter-derived numerical information is easily interpreted and may be immediately translated into therapeutic interventions. As it appears, for the time being, TEE may not yet replace the PA catheter as a monitoring modality.

In all the present three cases, the diagnosis had been established by TEE. It is conceivable that continuous TEE-monitoring may be beneficial in the early detection of the ICT, thus potentially increasing the likelihood of a better outcome. Despite the "pros and cons", TEE remains an invaluable diagnostic tool, whose capabilities cannot be overestimated.

Therapeutic modalities for acute intracardiac thrombosis include administration of intraoperative heparin and systemic thrombolytics, surgical or interventional radiological clot removal procedures, and catheter-directed thrombolytic therapy. It has been reported that systemic and catheter-directed thrombolysis (CDT) are potentially life-saving interventions in situations involving obstructing thrombus formation [16]. Precise, localized delivery of a thrombolytic agent reduces the risk for hemorrhagic complications in liver transplant recipients with significant coagulopathy, due to lower dosage used, and improved outcomes have been reported [17]. Successful use of recombinant tissue plasminogen activator (tPA) for intraoperative treatment of massive intracardiac and pulmonary thromboembolism have also been reported over the years in a few publications [18-20].

Hypercoagulability is considered a likely reason for left side thrombosis. Another important reason for the clot formation in the left heart chambers is a circulatory stasis. Reasons for stasis may be a very low $\mathrm{CO}$ (as in Case 1) or during inefficient CPR, or due to obstruction of the right-to-left circulation in cases of earlier right-sided thrombosis with pulmonary embolism. In all three cases here presented, the ICT manifested as a significant flow-obstructing mass, causing disastrous hemodynamic instability, with swift progression to circulatory arrest. No warning signs of potential thrombotic complication were observed. In every case, the patient demonstrated typical for ESLD coagulopathy, confirmed by TEG. Due to rapid development of circulatory shock, followed by a cardiac arrest, no attempt of 
thrombus removal was undertaken in the three cases, since such interventions were deemed futile under the circumstances.

\section{- CONCLUSIONS}

Massive ICT formation is a rare, life-threatening complication during liver transplantation, which remains mostly unpredictable and unpreventable, commonly leading to a poor outcome.

TEE is a valuable additional diagnostic and monitoring modality during liver transplant anaesthesia. The main limitation for TEE use in liver transplantation is that, unlike a PA catheter, it cannot provide continuous precise numerical information with alarm settings, that are immediately translatable into therapeutic interventions. TEE remains a definitive diagnostic tool for early detection of intracardiac thrombus formation and monitoring of myocardial function.

\section{CONCLICT OF INTEREST}

None to declare.

\section{REFERENCES}

1. Warnaar N, Molenaar IQ, Colquhoun SD, et al. Intraoperative pulmonary embolism and intracardiac thrombosis complicating liver transplantation: A systematic review. J Thromb Haemost 2008;6:297-302.

2. Gologorsky E1, De Wolf AM, Scott V, Aggarwal S, Dishart M, Kang Y. Intracardiac thrombus formation and pulmonary thromboembolism immediately after graft reperfusion in 7 patients undergoing liver transplantation. Liver Transpl. 2001 Sep;7(9):783-9.

3. Planinsic RM, Nicolau-Raducu R, Eghtesad B, Marcos A. Diagnosis and Treatment of Intracardiac Thrombosis During Orthotopic Liver Transplantation, Anesth Analg 2004;99:353-6

4. Protin C, Bezinover D, Kadry Z, Verbeek T. Case Report Emergent Management of Intracardiac Thrombosis during Liver Transplantation. Hindawi Publishing Corporation Case Reports in Transplantation 2016, Article ID 6268370:1-5

5. Dalia AA, Khan $H$, Flores AS. Intraoperative diagnosis of intracardiac thrombus during orthotopic liver transplantation with transesophageal echocardiography: A case series and literature review. Semin Cardiothorac Vascular Anesth2017;21:245-51.

6. Sogaard KK, Horvath-Puho E, Gronbaek H, Jepsen P, Vilstrup $H$, Sorensen HT. Risk of venous thromboembolism in patients with liver disease: a nationwide population based case-control study. Am J Gastroenterol 2009; 104: 96
7. Verbeek T, Stine J, Saner F, Bezinover D. Hypercoagulability in End-stage Liver Disease: Review of Epidemiology, Etiology, and Management, Transplantation Direct 2018;4: e403;

8. Bezinover D, Dirkmann D, Findlay J, Guta C, et al. Perioperative Coagulation Management in Liver Transplant Recipients. Transplantation 2018;102: 578-592

9. Porter TR, Shillcutt SK, Adams MS, et al. Guidelines for the use of Echocardiography as a monitor for therapeutic intervention in adults: A report from the American Society of Echocardiography. J Am Soc Echocardiogr 2015;28:40-56

10. Soong W, Sherwani SS, Ault ML, et al. United States practice patterns in the use of transesophageal echocardiography during adult liver transplantation. J Cardiothorac Vasc Anesth 2014; 28:635-9.

11. Schumann R, Mandell MS, Mercaldo N, et al. Anesthesia for liver transplantation in United States academic centers: Intraoperative practice. J Clin Anesth 2013;25:542-50.

12. WaxD, Torres A, Scher C, etal. Transesophageal echocardiography utilization in high-volume liver transplantation centers in the United States.J Cardiothorac Vasc Anesth 2008;22:811-3

13. Isaak RS, Kumar PA, Arora H. Pro: Transesophageal echocardiography should be routinely used for all liver transplant surgeries. J Cardiothorac Vasc Anesth2017;31:2282-6

14. Cywinski JB, Maheshwari K. Con: Transesophageal echocardiography is not recommended as a routine monitor for patients undergoing liver transplantation. J Cardiothorac Vasc Anesth 2017;31:2287-99

15. Piercy M, Mc Nicol L, Dinh DT, et al. Major complications related to the use of transesophageal echocardiography in cardiac surgery. J Cardiothorac Vasc Anesth2009;23:62-5

16. Kearon C, Akl EA, Comerota AJ, et al. Antithrombotic therapy for VTE disease: Antithrombotic therapy and prevention of thrombosis, American College of Chest Physicians Evidence-Based Clinical Practice Guidelines. Chest 2012;141(2Suppl):e419S-4

17. Piazza G, Hohlfelder B, Jaff MR, et al. A prospective, single-arm, multicenter trial of ultrasound-facilitated, catheter-directed, low-dose fibrinolysis for acute massive and submassive pulmonary embolism: The SEATTLE II study . JACC Cardiovasc Interv 2015;8:1382-92.

18. Jackson D, Botea A, Gubenko Y, et al. Successful intraoperative use of rTPA during liver transplantation complicated by massive intracardiac/pulmonary thrombosis. Anesth Analg 2006;102:724-8.

19. Kim S, DeMaria S Jr, Cohen E, Silvay G, Zerillo J. Prolonged Intraoperative Cardiac Resuscitation Complicated by Intracardiac Thrombus in a Patient Undergoing Orthotopic Liver Transplantation Seminars in Cardiothoracic and Vascular Anesthesia 2016, 20(3): 246-251

20. Boone J, Sherwani S, Herborn J, Patel K, MD, De Wolf A. The Successful Use of Low-Dose Recombinant Tissue Plasminogen Activator for Treatment of Intracardiac/Pulmonary Thrombosis During Liver Transplantation Anesth Analg, Feb 2011, Vol 112; 2: $319-321$ 